\title{
Erratum to: Macrophage M1 Plays a Positive Role in Aseptic Inflammation-Related Graft Loosening After Anterior Cruciate Ligament Reconstruction Surgery
}

\author{
Bin Song, ${ }^{1}$ Chuan Jiang, ${ }^{1}$ Huan Luo, ${ }^{1}$ Zhong Chen, ${ }^{1}$ Jingyi Hou, ${ }^{1}$ Yunfeng Zhou, ${ }^{1}$ Rui Yang, \\ Huiyong Shen, ${ }^{1,2}$ and Weiping $\mathrm{Li} \oplus^{1,2}$
}

Erratum to: Inflammation (2017)

https://doi.org/10.1007/s10753-017-0616-3

The original version of this article unfortunately contained a mistake. The first author's name on the published version of the article was wrong; Bing Song should be changed to Bin Song. With this, the original article was corrected and the correct name is now presented above.

The online version of the original article can be found at https://doi.org/ 10.1007/s10753-017-0616-3.

\footnotetext{
${ }^{1}$ Department of Orthopaedics, Sun Yat-sen Memorial Hospital, Sun Yatsen University, 107 West Yanjiang Road, Guangzhou, 510120, People's Republic of China

${ }^{2}$ To whom correspondence should be addressed at Department of Orthopaedics, Sun Yat-sen Memorial Hospital, Sun Yatsen University, 107 West Yanjiang Road, Guangzhou, 510120, People's Republic of China.E-mails: hyshen_sysmh@hotmail.com; 1wpsys@126.com
} 